\title{
Stability of Travelling Wave Solutions for Coupled Surface and Grain Boundary Motion
}

\author{
Margaret Beck * Zhenguo Pan ${ }^{\dagger}$ Brian Wetton ${ }^{\ddagger}$ \\ May 14, 2010
}

\begin{abstract}
We investigate the spectral stability of the travelling wave solution for the coupled motion of a free surface and grain boundary that arises in materials science. In this problem a grain boundary, which separates two materials that are identical except for their crystalline orientation, evolves according to mean curvature. At a triple junction, this boundary meets the free surfaces of the two crystals, which move according to surface diffusion. The model is known to possess a unique travelling wave solution. We study the linearization about the wave, which necessarily includes a free boundary at the location of the triple junction. This makes the analysis more complex than that of standard travelling waves, and we discuss how existing theory applies in this context. Furthermore, we compute numerically the associated point spectrum by restricting the problem to a finite computational domain with appropriate physical boundary conditions. Numerical results strongly suggest that the two-dimensional wave is stable with respect to both two- and three-dimensional perturbations.
\end{abstract}

Key words: Grain Boundary; Mean Curvature Motion; Surface Diffusion; Linear Stability; Spectrum; Free boundary.

*mabeck@math.bu.edu, Department of Mathematics and Statistics, Boston University, 111 Cummington St., Boston, MA 02215 USA

†matpz@nus.edu.sg, Department of Mathematics, National University of Singapore, 2 Science Drive 2, Singapore 117543

${ }^{\ddagger}$ corresponding author, wetton@math.ubc.ca, Department of Mathematics, University of British Columbia, Vancouver, B.C. Canada V6T 122 


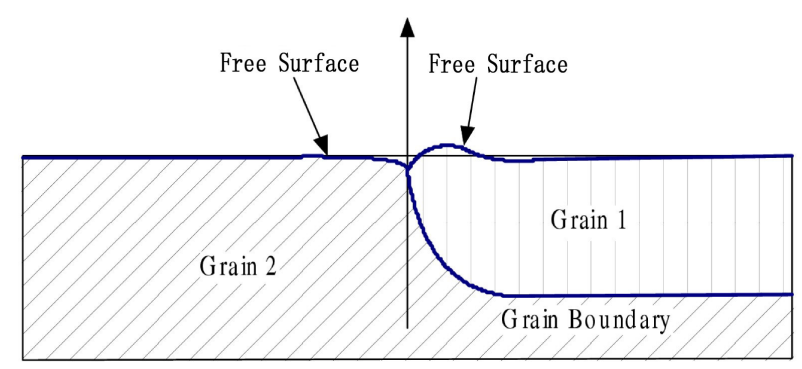

Figure 1: The quarter loop bi-crystal geometry.

\section{Introduction}

Many physical processes in material science and other fields can be approximately described by geometrical motion. For example, interfaces between crystal grains in an annealing process can be described by curvature motion in an idealized, isotropic setting. In two dimensional (2D) processes these interfaces meet at triple junctions at prescribed angles. A comprehensive introduction to grain boundary motion is given in [12]. This type of geometric curve network problem has attracted much interest in the literature, both analytical $[8,11]$ and numerical $[2,20]$. The statistics of grain sizes and its change with time is also of interest [1].

In this paper, we investigate the spectral stability of travelling waves in a model for coupled surface and grain boundary motion. This is an important phenomenon controlling the grain growth in materials processing and synthesis. The basic physics for this phenomenon were introduced in Dunn et. al. [9] in the so-called the "quarter loop" geometry shown in Figure 1. The grain boundary runs parallel to a free surface before it turns up and attaches to the upper free surfaces at a groove root. The point at which the three curves meet is often referred to as a triple junction. In certain applications, when heated at a specific temperature, the grain boundary can migrate to reduce the surface energy and to heal the orientation mismatch. This can lead to waves travelling at a constant speed, in which one grain grows at the expense of the other. Semi-explicit formulae of these travelling wave solutions can be found in $[14,15]$. The grain boundary curve in Figure 1 moves with curvature motion, that is with normal velocity equal to the local curvature. The free surface curves in Figure 1 move with surface 
diffusion. Because the quarter-loop problem in Figure 1 involves both curvature motion (second order parabolic) and surface diffusion (fourth order parabolic) we describe it as a mixed-order problem. Although the detailed composition of the material can be quite complex, a simple model composed of a grain boundaries separating components that are identical except for their crystalline orientation can help elucidate certain aspects of the way the material behaves. For example, these models give insight into a mixture of electrical steel and silicon that is an important component in transformers [10]. In this application, the properties of the material are closely related to the orientation of the grains.

In general, this process is three-dimensional. As suggested by Figure 1, we will make the standard assumption that the bi-crystal is uniform along the cross-sectional direction [16] leading to a two-dimensional model. Ultimately, one would like to understand the spectral stability of the travelling wave for the full three-dimensional mixed-order problem. Roughly speaking, this means linearizing the system about the $2 \mathrm{D}$ travelling wave and determining the spectrum of the resulting operator. If the spectrum, which consists of both continuous and point spectrum, lies in the left half of the complex plane, then the wave is said to be spectrally stable. In general, it is possible to analytically determine the location of the continuous spectrum, but difficult to do so for the point spectrum, also known as the eigenvalues. Therefore, we will compute the eigenvalues numerically. This will be done using the partial differential algebraic equation (PDAE) formulation of the problem proposed in [20] which has several advantages in this setting and is described in more detail below and in $\S 2$. In $\S 4$ and 5 , we will present numerical results that strongly suggest such two-dimensional travelling wave solutions for the mixed order problem shown in Figure 1 are stable to two- and threedimensional perturbations. This is the main contribution of this work. There is little analysis or computational work in the literature for this mixed-order problem in 3D. The work in this paper helps to fill that gap for this wellstudied process. See also [4] for recent computational work on this problem in $3 \mathrm{D}$.

In addition to this mixed-order problem, we also consider a simplified equal-order model, shown in Figure 2, that involves only grain boundary (curvature) motion. In this model, three grain boundaries meet at a triple junction and evolve by curvature motion. At the triple junction, the curves meet at the given angles $\theta$ shown. Far away from the triple junction, they run parallel to the horizontal direction. 


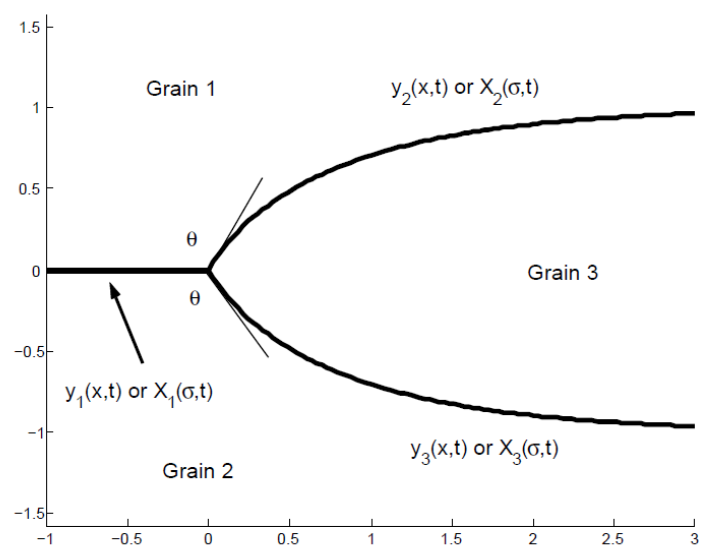

Figure 2: A curvature motion model.

Solving general geometric curve motion problems with junctions numerically has received a lot of recent attention [17, 24, 2, 3]. A number of numerical approaches were considered in $[25,26]$ applied to the quarterloop problem of interest in the current paper. The PDAE formulation was identified as having superior numerical properties during this study and was presented in [20]. The travelling wave solutions considered here were used to verify convergence of this method. The approach discovered independently in [2], although presented quite differently, has underlying similarities to our approach. This formulation is used in the present work for the numerical eigenvalue determination for the linearized problem around travelling waves.

A theoretical study of stability is most easily performed for problems written in Cartesian formulations. In that context, we must allow for perturbations not just of the wave but also of the location of the triple junction. As discussed in $\S 2$ below, this leads to the presence of a free boundary in the linearized problem, which can potentially create additional difficulties and new phenomenon in the analysis. Stability theory in the presence of free boundaries has been studied rigorously in $[6,7]$, and below we will discuss what these results imply about the current models. Our theoretical discussion is less rigorous for the PDAE formulation of the mixed-order problem, although we observe good agreement between theoretical predictions of the absolute spectrum and numerical results. 
Another issue we must deal with is that numerical calculation of the eigenvalues necessarily involves the truncation of the unbounded spatial domain to one of finite length, and one must be careful in choosing the boundary conditions so as not to create spurious eigenvalues. Rigorous results for stability problems in this context can be found in $[5,21,22]$, which we will use to determine the appropriate boundary conditions for this model.

The outline of the remainder of the paper is as follows. The equations of the models are described in detail in $\S 2$. We then present a theoretical discussion, mainly targeted at the simple, equal order problem, in $\S 3$. For this problem, there exists an explicit travelling wave solution in Cartesian coordinates. We recall here existing linear stability theory, including that involving a free boundary and domain truncation for numerical calculation of the point spectra. In $\S 4$, the PDAE method is used to investigate the stability to $2 \mathrm{D}$ perturbations of the travelling wave in the simplified model and the mixed-order problem. In $\S 5$ the analysis is extended to 3D perturbations of the mixed-order problem. Our results indicate that the travelling waves are stable in all these settings.

\section{Model Formulation}

\subsection{Simple, equal order grain boundary model}

The equations and junction conditions for the simple model shown in Figure 2 are described below first in terms of two-dimensional Cartesian coordinates. This Cartesian formulation is amenable to the theoretical discussion in $\S 3$ of the travelling waves we consider. An alternate formulation, the PDAE formulation, is subsequently described which is the basis of the numerical investigation of the point spectra.

To describe the simple curvature motion network problem shown in Figure 2 in terms of Cartesian coordinates, let $y_{i}(x, t), i=1,2,3$ be the vertical displacement of each curve. The location $x=q(t)$ of the junction must be introduced as an additional unknown. In this formulation, the problem is of free boundary type. Curvature motion of the three curves is described by

$$
\partial_{t} y_{i}=\frac{\partial_{x}^{2} y_{i}}{1+\left(\partial_{x} y_{i}\right)^{2}}
$$


with junction conditions

$$
\begin{gathered}
y_{1}(q(t), t)=y_{2}(q(t), t)=y_{3}(q(t), t) \\
\arctan \left[\partial_{x} y_{1}(q(t), t)\right]-\arctan \left[\partial_{x} y_{2}(q(t), t)\right]=\theta \\
\arctan \left[\partial_{x} y_{1}(q(t), t)\right]-\arctan \left[\partial_{x} y_{3}(q(t), t)\right]=-\theta
\end{gathered}
$$

and far field conditions

$$
y_{1}(-\infty, t)=0, \quad y_{2}(+\infty, t)=1, \quad y_{3}(+\infty, t)=-1 .
$$

This free-boundary, Cartesian formulation forms the basis of the discussion in $\S 3$.

The curves in the simple curvature motion network problem shown in Figure 2 can also be described by parametrized curves $X_{i}(\sigma, t)$ with parameter $\sigma \in[0, \infty)$ with $\sigma=0$ corresponding to the junction. Here, $X$ denotes a quantity with two components $(x, y)$ in $2 \mathrm{D}$ and three components $(x, y, z)$ in 3D. In this formulation, curves that are not single valued functions can be described. Such curves can be present in mixed order travelling waves as shown in Figure 7. An additional numerical advantage of this parametrized curve formulation is that the junction is a fixed boundary condition, not a free boundary one. For general parametrized curves in $2 \mathrm{D}$, the tangent $T$ and normal $N$ vectors and the curvature $\kappa$ can be calculated as follows

$$
\begin{aligned}
T & =\frac{\partial_{\sigma} X}{\left|\partial_{\sigma} X\right|} \\
N & =T^{\perp} \\
\kappa & =\frac{\partial_{\sigma}^{2} X \cdot N}{\left|\partial_{\sigma} X\right|^{2}}
\end{aligned}
$$

The correct normal motion is achieved when

$$
\partial_{t} X_{i} \cdot N_{i}=\kappa_{i}
$$

for $i=1,2,3$, where $\kappa_{i}$ and $N_{i}$ are computed from derivatives of $X_{i}$ with respect to $\sigma$ as indicated above. Notice that the tangential velocity away from the junction can be chosen arbitrarily. An additional condition to specify the parametrization with good numerical properties was identified in [20] and independently in [2]. Additional details about the formulation and alternative approaches to the problem can be found in these papers. The additional condition is

$$
\partial_{\sigma} X_{i} \cdot \partial_{\sigma}^{2} X_{i}=0
$$


This enforces that the parametrization remains a scaled arc length, although the scaling can change in time for finite length curves. This is seen by rewriting the condition above as

$$
\frac{1}{2} \partial_{\sigma}\left|\partial_{\sigma} X_{i}\right|^{2}=0
$$

The junction conditions for the parametrized formulation above are

$$
\begin{gathered}
X_{1}(0, t)=X_{2}(0, t)=X_{3}(0, t) \\
\frac{\partial_{\sigma} X_{1}(0, t)}{\left|\partial_{\sigma} X_{1}(0, t)\right|} \cdot \frac{\partial_{\sigma} X_{i}(0, t)}{\left|\partial_{\sigma} X_{i}(0, t)\right|}=\cos \theta \quad i=2,3
\end{gathered}
$$

and the far-field conditions are

$$
\begin{gathered}
X_{1}(\infty, t)=(-\infty, 0) \\
X_{2}(\infty, t)=(\infty, 1) \\
X_{3}(\infty, t)=(\infty,-1) \\
\left|\partial_{\sigma} X_{i}(\infty, t)\right|=1, \quad i=1,2,3
\end{gathered}
$$

The last condition fixes the parametrization $\sigma$ to be arc length. Note that $\sigma=+\infty$ represents the far-field for all curves in this formulation.

\subsection{Mixed-order, quarter loop model}

We now return to the mixed-order, quarter loop problem shown in Figure 1. Aspects of the model of this phenomenon described below were originally proposed in [18], and its present form was developed in [13] but rewritten in the PDAE formulation of parametrized curves developed in [20]. Let $X_{1}(\sigma, t)$ denote the grain boundary and $X_{2,3}(\sigma, t)$ denote the free surfaces to the left and right of the triple junction. The curves are parametrized with $\sigma \geq 0$ as above. Again $\sigma$ will remain arc length and $\sigma=0$ corresponds to the triple junction. A close-up of the junction is shown in Figure 3. Note that the numbering of the curves follows a different pattern than in the simple equal order model problem discussed above.

As mentioned above, the quarter loop geometry contains two types of motion: mean curvature motion for the grain boundary and motion by surface diffusion for the upper free surfaces. The normal velocity of the grain boundary is proportional to its curvature,

$$
\partial_{t} X_{1} \cdot N_{1}=A \kappa_{1}
$$




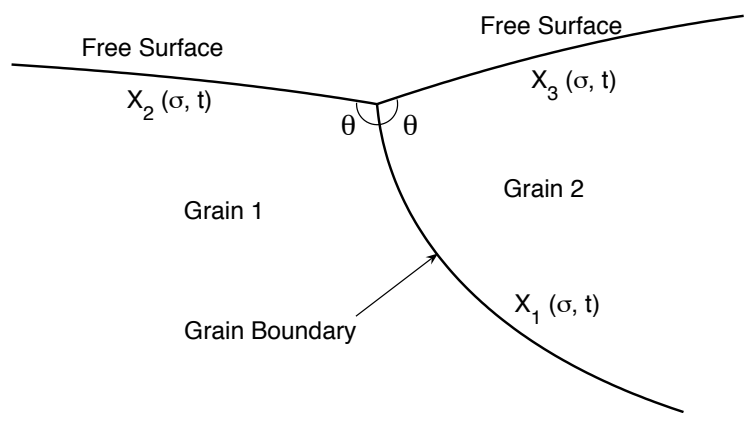

Figure 3: A labelled close-up of the junction in the mixed-order, quarter loop problem shown in Figure 1

which can be computed from derivatives of $X_{1}$ as in the simple equal order model above. $A$ is a positive physical constant. The normal velocities of the free surfaces undergoing surface diffusion are proportional to the surface Laplacian of curvature, which in 2D is $\partial_{s}^{2} \kappa$ where $s$ is arc length. In our formulation in which $\sigma$ is scaled arc length, this term can be written

$$
\partial_{s}^{2} \kappa=\frac{\partial_{\sigma}^{2} \kappa}{\left|\partial_{\sigma} X\right|^{2}}
$$

and so can be written simply in terms of derivatives of $X$ with respect to $\sigma$. For the infinite length curves of this model, $\sigma$ can remain arc length so the denominator above is unity. The motion of the free surfaces (upper curves) is then described by

$$
\partial_{t} X_{2,3} \cdot N_{i}=-B \partial_{\sigma}^{2} \kappa_{i}, \quad i=2,3
$$

where $B$ is a positive physical parameter. One can show that, by an appropriate rescaling of space and time, the constants $A$ and $B$ can be taken to be one [20]. Therefore, the model we will study is

$$
\begin{aligned}
& \partial_{t} X_{1} \cdot N_{1}=\kappa_{1} \\
& \partial_{t} X_{2} \cdot N_{2}=-\partial_{\sigma}^{2} \kappa_{2} \\
& \partial_{t} X_{3} \cdot N_{3}=-\partial_{\sigma}^{2} \kappa_{3} .
\end{aligned}
$$


In addition to equations (8), one must also include

$$
\partial_{\sigma} X_{i} \cdot \partial_{\sigma}^{2} X_{i}=0, \quad i=1,2,3 .
$$

to fix the parametrization as discussed above. Junction conditions are listed below:

$$
\begin{gathered}
X_{1}(0, t)=X_{2}(0, t)=X_{3}(0, t) \\
\partial_{s} X_{1} \cdot \partial_{s} X_{i}=\cos \theta \quad i=2,3 \\
\kappa_{2}=-\kappa_{3} \\
\partial_{s} \kappa_{2}=\partial_{s} \kappa_{3} .
\end{gathered}
$$

where derivatives in arc length $s$ can be written as scaled derivatives in $\sigma$ as discussed above. The first equation in (10) expresses that the curves meet at a common junction. The second equation, Young's Law, represents a balance of surface tensions. Here $\theta=\pi / 2+\arcsin (m / 2)$ denotes the angle between the grain boundary and the exterior surfaces and $m=\gamma_{\text {grain }} / \gamma_{\text {exterior }} \geq 0$ is a constant measuring the relative surface tensions $\gamma$ between them. These junction conditions are modelled in the simple, equal order model above. The remaining conditions are necessary to complete the specification of the fourth order, free surface problem. The third condition in (10) reflects the continuity of the surface chemical potentials, and the last condition represents the balance of mass flux. Again, the reader can refer to [13] for a discussion of the model equations (8) and junction conditions (10) and to [20] for a discussion of the parametrization constraint (9). In this paper, we consider the linear stability (to 2D and 3D perturbations) of 2D travelling waves for this system known in the literature. To consider 3D perturbations, a 3D version of the problem must be considered. The technical details of this case are left to $§ 5$. Far field conditions for the travelling wave are

$$
\begin{gathered}
X_{1}(\infty, t)=(\infty,-H) \\
X_{2}(\infty, t)=(-\infty, 0) \\
X_{3}(\infty, t)=(\infty, 0) \\
\partial_{\sigma} x_{i}(\infty, t)=1, \quad i=1,2,3 \\
\partial_{\sigma} y_{i}(\infty, t)=0, \quad i=2,3
\end{gathered}
$$

where as $\left(x_{i}, y_{i}\right)$ are the components of the curve $X_{i}$ and $H$ is a specified grain thickness in the far field that enters as a parameter in the travelling 
wave. The first four conditions above parallel the ones for the simple, equal order model and the last condition is needed for asymptotic flatness of the higher order free surface curves.

Existence of the travelling wave was proven in [14] for $m \lesssim 0.92$ and in [15] for all $m \in[0,2)$. In these papers, implicit formulae for the travelling waves are also given. Recall that $m$ is a parameter in Young's Law that determines the angles between the free surfaces and the grain boundary. For $1.81 \lesssim m<2$ the wave is no longer single-valued (for $y$ as a function of $x$ ) and so it is referred to as a "non-classical travelling wave." An example of such a solution is shown in Figure 7.

\section{$3 \quad$ Spectral stability of travelling waves}

This section contains a theoretical discussion of travelling wave stability in the context of geometric motion with junctions. Some rigorous statements can be made on the simple, equal-order model of grain boundary motion shown in Figure 2. This problem is particularly amenable to analysis because it possesses an explicit travelling wave solution in terms of Cartesian coordinates, which is presented below. We discuss the linearization about the wave and related stability theory, including that involving a free boundary and domain truncation. Some less rigorous statements are made about the mixed-order problem, motivated by the discussion of the equal-order problem. The discussion of this section indicates the stability of travelling waves up to the possible presence of point spectra, which are investigated numerically in later sections.

\subsection{Simple, equal-order model}

The simple model (1) with junction (2) and far-field (3) conditions has an explicit travelling wave solution given by

$$
\begin{aligned}
& \bar{y}_{1}(\xi)=0 \\
& \bar{y}_{2}(\xi)=1-\frac{1}{c} \arcsin \left[\sin (c) e^{-c \xi}\right] \\
& \bar{y}_{3}(\xi)=-1+\frac{1}{c} \arcsin \left[\sin (c) e^{-c \xi}\right],
\end{aligned}
$$

where the wave speed is $c=\pi-\theta$, the junction location is given by $q(t)=c t$, and the moving coordinate $\xi$ is defined by $\xi=x-c t$. For a derivation of 
this solution see [19].

We need to allow for a perturbation not just of the grain boundaries but also of the junction location. This is in contrast to studying the stability of a travelling wave without the presence of a junction. In that case, if the original PDE is translation invariant, then any fixed translate of the travelling wave will also be a solution. This manifests itself in the stability problem through the presence of a zero eigenvalue with associated eigenfunction given by the derivative of the wave. Any changes in the speed of the travelling wave, and hence changes in the location of the interface, can be captured by the behaviour of perturbations. In the present context, we should still allow for perturbations that are simply translates of the underlying wave. To do this, we need to allow for a change in the junction location $q(t)$. Otherwise, there would be a mismatch in the junction conditions between the perturbation and the underlying wave. We will see this explicitly, below.

Write the junction location as

$$
q(t)=c t+p(t)
$$

and define a new coordinate $\tilde{x}=x-q(t)$. We proceed below with equations for the curves $y_{1}(\tilde{x}, t)$, dropping the tilde's on the $x$ variables. Following $[6,7]$, we write the perturbation of the wave as

$$
y_{i}(x, t)=\bar{y}_{i}(x)+p(t) \partial_{x} \bar{y}_{i}(x)+v_{i}(x, t), \quad i=1,2,3 .
$$

Note that, to leading order in $p$, this is equivalent to

$$
y_{i}(x, t)=\bar{y}_{i}(\xi)+v_{i}(x, t) .
$$

The motivation for this can be understood as follows. It is advantageous to work in the coordinates $(x, t)$ because then the junction becomes fixed at $x=0$. However, simply defining

$$
y_{i}(x, t)=\bar{y}_{i}(x)+v_{i}(x, t)
$$

would introduce a term of the form $\dot{p} \partial_{x} \bar{y}_{i}$ into the linearized equation. Using the Ansatz (13) allows one to remove this term at the expense of complicating the conditions at the junction.

Inserting (13) into (1)-(2) and retaining only the terms that are linear in $v_{i}$ and $p$, we obtain

$$
\partial_{t} v_{i}=\left(\frac{1}{1+\left(\partial_{x} \bar{y}_{i}\right)^{2}}\right) \partial_{x}^{2} v_{i}+\left(c-\frac{2 \partial_{x} \bar{y}_{i} \partial_{x}^{2} \bar{y}_{i}}{\left(1+\left(\partial_{x} \bar{y}_{i}\right)^{2}\right)^{2}}\right) \partial_{x} v_{i}=: L_{i} v_{i}
$$


for $i=1,2,3$, with the junction conditions

$$
\begin{aligned}
\tan (c) p(t)= & v_{1}(0, t)-v_{2}(0, t)=-v_{1}(0, t)+v_{3}(0, t) \\
\tan (c)+\tan (\theta)= & {[1-\tan (\theta) \tan (c)] \partial_{x} v_{1}(0, t)-\partial_{x} v_{2}(0, t) } \\
& -2 c \sec ^{2}(c) \tan (c) p(t) \\
\tan (c)+\tan (\theta)= & {[-1+\tan (\theta) \tan (c)] \partial_{x} v_{1}(0, t)+\partial_{x} v_{3}(0, t) } \\
& -2 c \sec ^{2}(c) \tan (c) p(t)
\end{aligned}
$$

and asymptotic condition

$$
v_{1}(-\infty, t)=v_{2}(+\infty, t)=v_{3}(+\infty, t)=0 .
$$

Note that we can combine the equations in (15) to obtain

$$
\begin{aligned}
0= & -2 v_{1}(0, t)+v_{2}(0, t)+v_{3}(0, t) \\
\tan (c)+\tan (\theta)= & {[1-\tan (\theta) \tan (c)] \partial_{x} v_{1}(0, t)-\partial_{x} v_{2}(0, t) } \\
& -2 c \sec ^{2}(c)\left[v_{1}(0, t)-v_{2}(0, t)\right] \\
\tan (c)+\tan (\theta)= & {[-1+\tan (\theta) \tan (c)] \partial_{x} v_{1}(0, t)+\partial_{x} v_{3}(0, t) } \\
& +2 c \sec ^{2}(c)\left[v_{1}(0, t)-v_{3}(0, t)\right]
\end{aligned}
$$

Below, we will use the fact that, since $c$ and $\theta$ are fixed, (17) is linear in the six variables $\left(v_{1}(0, t), v_{2}(0, t), v_{3}(0, t), \partial_{x} v_{1}(0, t), \partial_{x} v_{2}(0, t), \partial_{x} v_{3}(0, t)\right)$.

In order to rigorously analyze the spectral stability of the wave, one would need to verify several things about the system (14) - (16). In particular, one would need to formulate the linear operator in (14) as acting on an appropriate function space that incorporates the boundary and asymptotic conditions (15) and (16). In addition, one would need to calculate the spectrum of the operator on that space, or at least prove that the spectrum was contained entirely within the left half of the complex plane. Since here our focus is primarily the numerical computation of the spectrum, we will be content to simply indicate why (14) - (16) is a reasonable way to formulate the stability problem.

In $[6,7]$ the stability of travelling waves in the presence of free boundaries was analyzed rigorously. In those works, the authors use an Ansatz of the form (13) to fix the location of the boundary at the expense of converting a linear condition at the boundary to a fully nonlinear one. They prove that this leads to a well defined spectral problem and use it to rigorously prove the stability of travelling waves with free boundaries in several applications. Our 
setting, (1)-(2), is slightly more complicated because the equations themselves are more complex and the conditions at the free boundary are, at the outset, nonlinear. However, we expect that a similar analysis would apply.

When studying unbounded linear operators, there are several ways to characterize different elements of the spectrum. For linear operators on unbounded domains, such as the one in equation (14), the most useful way is to divide the spectrum into the so-called continuous, or essential, spectrum and the point spectrum, or eigenvalues. The point spectrum is defined to be the set of all isolated eigenvalues of finite multiplicity, and the essential spectrum is its complement within the spectrum. The reason this is a useful characterization is because in general one can explicitly compute the essential spectrum using the asymptotic limits of the operator [21, 22].

For example, as $x \rightarrow \pm \infty$, equation (14) becomes

$$
\partial_{t} v_{i}=\partial_{x}^{2} v_{i}+c \partial_{x} v_{i}=: L_{\infty} v_{i}, \quad i=1,2,3 .
$$

The associated eigenvalue equation is

$$
\lambda v=L_{\infty} v
$$

which has explicit solutions given by exponentials. A bounded solution exists only when $\lambda=-k^{2}+i c k$ for some $k \in \mathbb{R}$, and therefore the spectrum of $L_{\infty}$ is given exactly by this set. The results in [21] then imply that the essential spectrum associated to (14)-(16) is given by

$$
\Sigma_{\mathrm{ess}}=\left\{\lambda=-k^{2}+i c k: k \in \mathbb{R}\right\} .
$$

Determining the point spectrum is, in general, much more difficult. In certain situations, for example if the equation has conserved quantities or some type of fast-slow structure, one can analytically determine the eigenvalues or prove that they all lie in the left half of the complex plane. For details, we refer to the review [21] and, for the situation involving free boundaries, [6]. Here this is not the case, and so we will compute the eigenvalues numerically. Eigenvalues correspond to values of $\lambda \in \mathbb{C}$ for which the system

$$
\lambda v_{i}=L_{i} v_{i}, \quad i=1,2,3
$$

has a solution that satisfies (15)-(16). One can explicitly check that, for $\lambda=0, v_{i}=\delta \partial_{x} \bar{y}_{i}, i=1,2,3$, is an eigenfunction with $p(t)=-\delta$. As discussed above, this is expected and related to translations of the underlying wave. 
Another way to think of the linearized problem (14)-(16) is in terms of the Fredholm properties of the linear operator. Since on each half line the operators $L_{i}$ are just convection-diffusion operators, one can verify that system (20) for $i=1,2,3$ has Fredholm index three for values of $\lambda$ to the right of the essential spectrum. A bordering lemma can then be used to show that, after the addition of the boundary conditions at the junction, (15), the Fredholm index becomes zero. Thus, to understand the spectral stability of the traveling waves, one really need only look for eigenvalues to the right of the curve (19). For a discussion of the relationship between the Fredholm and spectral properties of a linear operator, see [21, §3.3]. A bordering lemma can be found in [23, Lemma 3.5].

Because any numerical calculations necessarily take place on a finite computational domain, one must truncate the domain to $(-L, 0)$ for $v_{1}$ and $(0, L)$ for $v_{2,3}$ and impose appropriate boundary conditions at $\pm L$. (Note that the junction conditions at zero remain as in equation (15).) In general, one needs to be careful when imposing artificial conditions at the ends of the finite domain, as spurious eigenvalues can be created. A rigorous study of domain truncation in the context of stability was conducted in [22]. In that work the authors did not consider situations involving a free boundary. However, equation (17) shows how the free boundary can be eliminated from conditions at the junction. Thus, the framework of [22] is applicable to the current setting, and so we now recall some of their results.

In [22], the authors consider a certain class of operators $\mathcal{L}$, including for example that which one would obtain when linearizing a system of reactiondiffusion equations around a travelling wave, defined on the infinite spatial domain $\mathbb{R}$. For the truncated domain $(-L, L)$, the potential boundary conditions at $\pm L$ were divided into two categories: periodic and separated. Periodic implies that any eigenfunction must satisfy $v(-L)=v(L)$, and separated includes Dirichlet, $v( \pm L)=0$, and Neumann, $v^{\prime}( \pm L)=0$, conditions. The authors were interested in studying how the point spectrum of $\mathcal{L}$ on the truncated domain, $\Sigma_{L}$, with a given set of boundary conditions was related to the spectrum on the infinite domain, $\Sigma$. They found that, for periodic conditions, in the limit $L \rightarrow \infty, \Sigma_{L}$ accurately approximates both the point and essential spectrum of $\mathcal{L}$. However, for separated boundary conditions, although $\Sigma_{L}$ will capture the point spectrum of $\mathcal{L}$ in the limit $L \rightarrow \infty$, additional eigenvalues can also be created. These spurious eigenvalues are not necessarily relevant for the stability on the infinite domain and are created by the boundary conditions. Thus, in general, it is better to use periodic 
conditions when numerically computing point spectra.

However, it is not possible for us to use periodic boundary conditions for (20). This is due to the presence of the triple junction. Condition (15), which any eigenfunction must satisfy, is effectively a separated boundary condition imposed at one end of the domain $(x=0)$ for each of the functions $v_{i}$. Therefore, periodic boundary conditions don't really make sense in this context. Since we will necessarily use separated boundary conditions, the most natural conditions to impose at $\pm L$ are therefore the physical conditions

$$
v_{1}(-L)=0, \quad v_{2,3}(+L)=0,
$$

which correspond to the asymptotic condition (16). We must check that these boundary conditions do not create any spurious eigenvalues, and in [22, §4.3] a condition is derived that allows one to do this. In order to explain this condition, we must reformulate the eigenvalue problem (20).

For notational clarity, fix $v=v_{i}$ and $\bar{y}_{i}=\bar{y}$, for $i=1,2,3$. We can write (20) as

$$
\frac{d}{d x} V=\mathcal{A}(x, \lambda) V, \quad V=\left(\begin{array}{c}
v \\
v_{x}
\end{array}\right)
$$

where

$$
\mathcal{A}(x, \lambda)=\left(\begin{array}{cc}
0 & 1 \\
\lambda\left(1+\bar{y}_{x}^{2}(x)\right) & -\left[c\left(1+\bar{y}_{x}^{2}(x)\right)-\frac{2 \bar{y}_{x}(x) \bar{y}_{x x}(x)}{1+\bar{y}_{x}^{2}(x)}\right]
\end{array}\right),
$$

and we note that

$$
\mathcal{A}^{ \pm}(\lambda):=\lim _{x \rightarrow \pm \infty} \mathcal{A}(x, \lambda)=\left(\begin{array}{cc}
0 & 1 \\
\lambda & -c
\end{array}\right)
$$

This formulation is often referred to as a spatial dynamical system, since it is an ODE in the spatial variable $x$. In addition to satisfying the junction condition, an eigenfunction $V$ must decay to zero as $x \rightarrow \pm \infty$. This can be related to the asymptotic matrices $\mathcal{A}^{ \pm}$, which are equal due to the fact that only derivatives of the underlying wave appear in the linearized problem. The eigenvalues of this matrix are given by $\nu_{s}(\lambda)=\left[-c-\sqrt{c^{2}+4 \lambda}\right] / 2$ and $\nu_{u}(\lambda)=\left[-c+\sqrt{c^{2}+4 \lambda}\right] / 2$. The subscripts stand for stable and unstable and are due to the fact that, for $\lambda>0, \operatorname{Re} \nu_{s}(\lambda)<0$ and $\operatorname{Re} \nu_{u}(\lambda)>0$. These eigenvalues are often referred to as spatial eigenvalues to distinguish 
them from the temporal eigenvalues $\lambda$. The associated stable and unstable subspaces are

$$
E^{s}(\lambda)=\left(\begin{array}{c}
1 \\
\nu_{s}(\lambda)
\end{array}\right), \quad E^{u}(\lambda)=\left(\begin{array}{c}
1 \\
\nu_{u}(\lambda)
\end{array}\right)
$$

In order for $V$ to decay to zero it would need to be asymptotic to $E^{s}$ as $x \rightarrow+\infty$ and asymptotic to $E^{u}$ as $x \rightarrow-\infty$.

As shown in [22], spurious eigenvalues can be created by the boundary conditions in the following way. Let $Q=(0,1)^{T}$. Any function $V$ that satisfies the Dirichlet boundary conditions must satisfy $V( \pm L) \in \operatorname{span}\{Q\}$. If $Q$ is parallel to either $E^{s}\left(\lambda_{*}\right)$ or $E^{u}\left(\lambda_{*}\right)$ for some $\lambda_{*}$, then the truncated eigenvalue problem can have a solution that actually becomes unbounded near $\pm L$ as $L \rightarrow \infty$. The reason for this is that, when $Q$ is parallel to either eigendirection, the function $V$ can satisfy the boundary conditions but lie entirely in the stable subspace at $-L$, or the unstable subspace at $+L$. Thus, even though $\lambda_{*}$ appears to be an eigenvalue, $V$ would have the wrong asymptotic behaviour as $L \rightarrow \infty$. Conversely, if $Q$ is not parallel to either subspace, one can use this fact to construct true eigenfunctions for the eigenvalue $\lambda_{*}$.

One can see explicitly that $Q$ is not parallel to $E^{s}$ or $E^{u}$ for any value of $\lambda$, and so the physical Dirichlet boundary conditions (21) will not create spurious eigenvalues. Note, however, that if one were to use Neumann boundary conditions, which correspond to $\tilde{Q}=(1,0)^{T}$, then this condition would be violated at $\lambda=0$. In $\S 4$, we will see that the zero eigenvalue indeed appears to have a higher multiplicity if one uses Neumann boundary conditions.

We conclude this section with a few remarks on the so-called absolute spectrum, which is defined in terms of the asymptotic limits of the operator. See [22, Definition 3.5] and $\S 3.2$ for more details. On unbounded domains, the absolute spectrum is not actually part of the spectrum. However, it can provide useful information related to the spectrum. In particular, for separated boundary conditions, the spectrum of the truncated operator $\Sigma_{L}$ will approximate the absolute spectrum, in addition to the true point spectrum, as $L \rightarrow \infty$. One can explicitly compute the absolute spectrum for (20) and see that it is $\left(-\infty,-c^{2} / 4\right]$. This is why, in the numerical calculations of $\S 4$, one sees approximations of this interval in addition to the eigenvalue zero. 


\subsection{Mixed-order, quarter loop problem}

It would be much more difficult to analyze the mixed-order problem with the same rigour as the equal-order problem above. In this work, more of the burden is placed on the numerical calculations in $\S 4$ and 5. However, we can follow formally the steps above and predict an absolute spectra in the finite domain that corresponds to that seen in the numerical computations.

Here, we consider the problem in 3D but linearized about the 2D travelling wave. We can formally consider the problem in the far field in terms of parametrized curves $y(x, z, t)$ (recall that near the junction, the travelling wave may not be single-valued). As above, we replace $x$ with a coordinate moving with the travelling wave speed but retain the same name $x$ below. As $x \rightarrow \pm \infty$

$$
\partial_{t} v=L_{\infty} v:=-\Delta \Delta v+c \partial_{x} v
$$

where $v$ is the linear perturbation to the travelling wave $\bar{y}$ in the moving frame with travelling wave speed $c$ and the biharmonic term $-\Delta \Delta=-\left(\partial_{x}^{2}+\partial_{z}^{2}\right)^{2}$ is the linearization of surface diffusion at a flat interface. This equation is the analogue of (18) above.

The associated eigenvalue equation is

$$
\lambda v=L_{\infty} v
$$

which as above has explicit solutions given by exponentials:

$$
\Sigma_{\mathrm{ess}}=\left\{\lambda=-\left(k^{2}+\omega^{2}\right)^{2}+i c k: k, \omega \in \mathbb{R}\right\} .
$$

Here, we introduce the wavenumber $\omega$ for perturbations in the $z$ direction that will appear as a parameter in the stability calculations in $\S 5$.

The absolute spectra which corresponds to this problem is not known analytically even in $2 \mathrm{D}(\omega=0)$. However, the absolute spectra of the $2 \mathrm{D}$ case

$$
\lambda v=-\partial_{x}^{4} v+c \partial_{x} v
$$

can be partially dealt with analytically and investigated numerically using [22, Definition 3.5]. We briefly review this for later comparison with the numerical results in $\S 4.3$.

The absolute spectrum is defined in terms of the roots of the characteristic equation associated with (26), given by

$$
\nu^{4}-c \nu+\lambda=0
$$




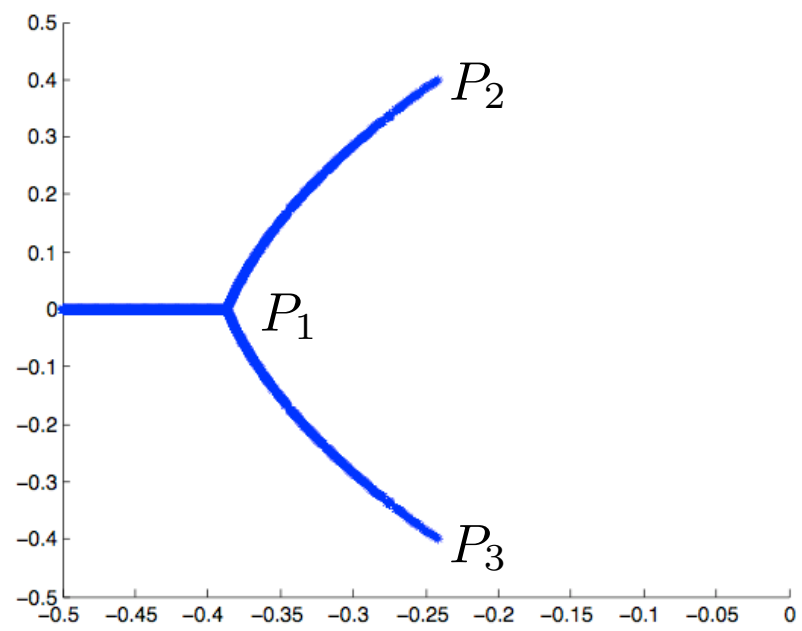

Figure 4: Numerical computation of the absolute spectra of (26) for $c=1$.

If the four spatial eigenvalues, which are simply roots of this equation, are ordered so that

$$
\operatorname{Re}\left(\nu_{1}(\lambda)\right) \leq \operatorname{Re}\left(\nu_{2}(\lambda)\right) \leq \operatorname{Re}\left(\nu_{3}(\lambda)\right) \leq \operatorname{Re}\left(\nu_{4}(\lambda)\right)
$$

then the absolute spectrum is defined as

$$
\Sigma_{\mathrm{abs}}=\left\{\lambda: \operatorname{Re}\left(\nu_{2}(\lambda)\right)=\operatorname{Re}\left(\nu_{3}(\lambda)\right)\right\} .
$$

For $c=-1$, the numerically computed absolute spectrum of (26) is shown in Figure 4.

Although it is difficult to analytically determine the roots of (27) - formulas exist for roots of a quartic but they are very complicated - one can calculate the boundary of the absolute spectrum, which are the points labeled $P_{1,2,3}$ in Figure 4.

Assume that $\lambda=\lambda_{\mathrm{r}}+\mathrm{i} \lambda_{\mathrm{i}}$ and $\nu=a+\mathrm{i} b$. Equating real and imaginary parts in the fourth order polynomial (27), we obtain

$$
\begin{aligned}
& f_{1}\left(a, b ; \lambda_{\mathrm{r}}, \lambda_{\mathrm{i}}\right)=a^{4}-6 a^{2} b^{2}+b^{4}-c a+\lambda_{\mathrm{r}}=0 \\
& f_{2}\left(a, b ; \lambda_{\mathrm{r}}, \lambda_{\mathrm{i}}\right)=4 a^{3} b-4 a b^{3}-c b+\lambda_{\mathrm{i}}=0 .
\end{aligned}
$$

At any point in the absolute spectrum, there will be two solutions to this equation given by $(a, b)$ and $(a, \tilde{b})$. Thus, it will not be possible to write 
$b=b(a)$ near those points; i.e. the conditions of the implicit function will fail. Therefore, to find these points, we just need to find where

$$
\partial_{b} f_{1}=-12 a^{2} b+4 b^{3}=0, \quad \partial_{b} f_{2}=4 a^{3}-12 a b^{2}-c=0 .
$$

The solution is given by $\left(a_{1}, 0\right)$ and $\left(a_{2,3}, b_{2,3}\right)$, where

$$
a_{1}=3\left(\frac{c}{4}\right)^{4 / 3}, \quad a_{2,3}=-\left(\frac{c}{32}\right)^{1 / 3}, \quad b_{2,3}= \pm \sqrt{3}\left(\frac{c}{32}\right)^{1 / 3} .
$$

Thus, we see that

$$
P_{1}=-\frac{21}{100}\left(\frac{5 c^{2}}{2}\right)^{2 / 3}, \quad P_{2,3}=-\frac{3 c}{8}\left(\frac{c}{4}\right)^{1 / 3} \pm \mathrm{i} \frac{3 \sqrt{3} c}{8}\left(\frac{c}{4}\right)^{1 / 3} .
$$

This absolute spectrum is seen approximately in the finite difference numerical computations in $\S 4.3$ below.

\section{Numerical Results in 2D}

In this section, we compute the spectrum numerically for travelling waves both for the simple, equal-order problem and the mixed-order, quarter loop problem. The discussion in $\S 3$ addressed the continuous spectra for these problems. The numerical results in this section are done primarily to rule out possible unstable point spectra. However, the results also confirm the predictions of the theory on the absolute spectra which for the mixed-order problem are not completely rigorous. The linearization and numerical approximation procedure is discussed below for the equal-order model which is less complex but representative.

\subsection{Linearization and numerical approximation of the simple, equal-order model}

We describe here the linearization and numerical approximation of the spectra for the PDAE formulation of the equal-order model given in $\S 2.1$. Let

$$
\bar{X}_{i}=\left(\bar{x}_{i}(\sigma), \bar{y}_{i}(\sigma)\right)
$$

represent the parametrized travelling wave solution in the moving frame $\tilde{x}=x-c t$ with wave speed $c=\pi-\theta$. This parametrized solution is obtained numerically by converting the Cartesian formulation (12) to a curve 
with parameter $\sigma$ that is approximately arc-length. Starting at the junction, points are determined that both lie on the travelling wave and are the given, fixed resolution distance $h=\Delta \sigma$ apart.

System (4) and (5) in the moving frame becomes

$$
\begin{aligned}
& \partial_{t} X_{i} \cdot N_{i}=\kappa_{i}-C \cdot N_{i} \\
& \partial_{\sigma} X_{i} \cdot \partial_{\sigma}^{2} X_{i}=0
\end{aligned}
$$

for $i=1,2,3$ where $C=(c, 0)$. Obviously, $\bar{X}$ defined by (29) is a steady state solution of above system.

To analyze the linear stability of the travelling wave solution, we consider a linear perturbation $\hat{X}_{i}$ to the steady state $\bar{X}_{i}$, i.e.,

$$
X_{i}=\bar{X}_{i}+\epsilon \hat{X}_{i}
$$

and linearize system (30) around $\bar{X}$ to get a linear system. We begin with the linearization of

$$
\left|\partial_{\sigma} X\right| \approx 1+\epsilon \partial_{\sigma} \bar{X} \cdot \partial_{\sigma} \hat{X}
$$

where the fact that the travelling wave parametrization is arc length is used. The linearization of the normal $N$ is thus

$$
N \approx \bar{N}+\epsilon\left(\partial_{\sigma} \hat{X}^{\perp}-\left(\partial_{\sigma} \bar{X} \cdot \partial_{\sigma} \hat{X}\right) \bar{N}\right)
$$

and curvature

$$
\kappa=\frac{\partial_{\sigma}^{2} X \cdot N}{\left|\partial_{\sigma} X\right|^{2}} \approx \bar{\kappa}+\epsilon\left(\partial_{\sigma}^{2} \hat{X} \cdot \bar{N}-3 \partial_{\sigma} \bar{X} \cdot \partial_{\sigma} \hat{X} \bar{\kappa}+\partial_{\sigma}^{2} \bar{X} \cdot \partial_{\sigma} \hat{X}^{\perp}\right)
$$

Inserting these linear expressions in (30) gives

$$
\begin{aligned}
\partial_{t} X_{i} \cdot \bar{N}_{i}= & \partial_{\sigma}^{2} X_{i} \cdot \bar{N}_{i}-3 \partial_{\sigma} \bar{X}_{i} \cdot \partial_{\sigma} X_{i} \bar{\kappa}_{i}+\partial_{\sigma}^{2} \bar{X}_{i} \cdot \partial_{\sigma} X_{i}^{\perp}- \\
& C \cdot\left(\partial_{\sigma} X_{i}^{\perp}-\left(\partial_{\sigma} \bar{X}_{i} \cdot \partial_{\sigma} X_{i}\right) \bar{N}_{i}\right) \\
0= & \left(\partial_{\sigma} \bar{X}_{i} \cdot \partial_{\sigma}^{2} X_{i}\right)+\left(\partial_{\sigma} X_{i} \cdot \partial_{\sigma}^{2} \bar{X}_{i}\right)
\end{aligned}
$$

for $i=1,2,3$. Here we have dropped the hats that indicate the $O(\epsilon)$ terms. The associated eigenvalue problem is given by replacing the time derivative $\partial_{t} X_{i}$ in the first equation of (31) by $\lambda X_{i}$. The junction conditions (6) can be linearized to give boundary conditions for this problem at $\sigma=0$. Boundary conditions that are homogeneous versions of (7) are applied at a finite 

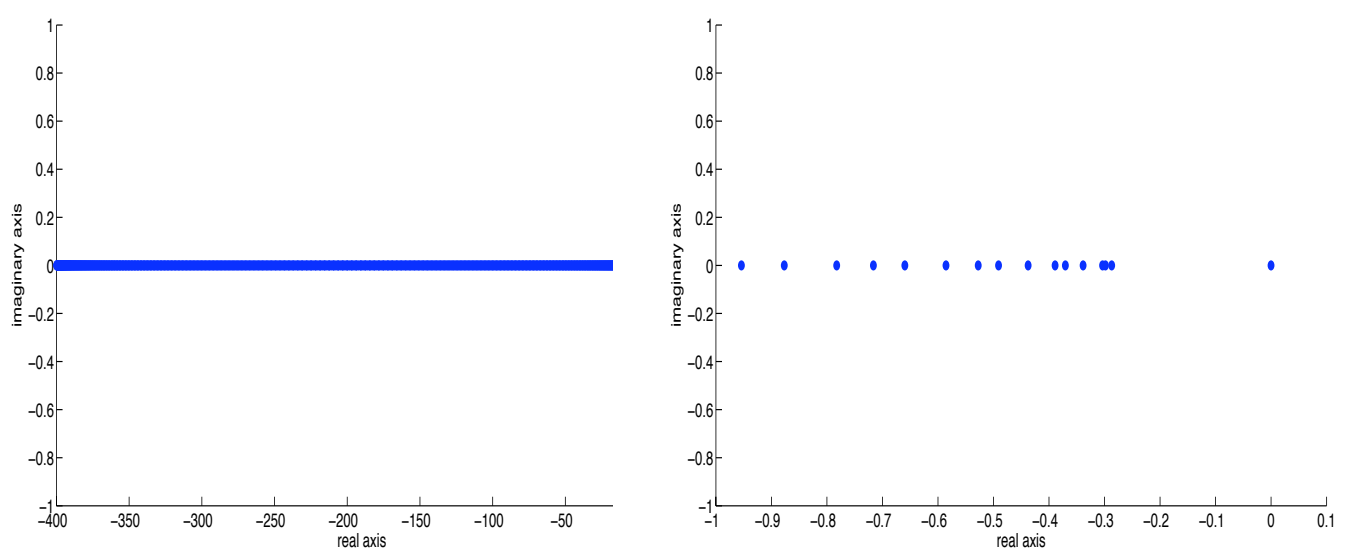

Figure 5: Approximate eigenvalue distribution for the equal order model problem. The computational domain size is $L=20$, discretization $h=$ $\Delta \sigma=0.1$ and the angles are equal $\theta=2 \pi / 3$. The right picture is a close look of the left picture near origin.

distance in arc length $\sigma=L$ from the junction. In (31) derivatives in $\sigma$ of the perturbation are approximated by finite differences and derivatives of the base solution can be found analytically. We now have a large, generalized eigenvalue problem. It is generalized because $\lambda$ does not appear in the constraint equations nor in the approximation of the linearization of the junction conditions (6). Numerical calculation of the eigenvalues for the discrete problem above are done using MATLAB's eig routine. The results are discussed below.

\subsection{Numerical results for the simple, equal-order prob- lem}

The eigenvalues of the generalized boundary value problem that corresponds to the equal order model derived above are approximated computationally. The distribution of the eigenvalues is shown in Figure 5 for a domain size of $L=20$, discretization $h=\Delta \sigma=0.1$ and equal angles $\theta=2 \pi / 3$. As expected, there is a single zero eigenvalue with associated eigenfunction given by the derivative of the travelling wave which corresponds to its translation

invariance. This eigenvalue is the only member of the point spectrum found 


\begin{tabular}{|c|c|}
\hline$L=20$ fixed & \\
$h$ & $\lambda$ \\
\hline 0.12 & -0.2872 \\
0.06 & -0.2870 \\
0.03 & -0.2869 \\
0.015 & -0.2868 \\
\hline
\end{tabular}

\begin{tabular}{|c|c|}
\hline$h=0.1$ fixed & \\
$L$ & $\lambda$ \\
\hline 7.5 & -0.3404 \\
15 & -0.2948 \\
30 & -0.2809 \\
60 & -0.2764 \\
\hline
\end{tabular}

Table 1: Real part of the leading non-zero approximate eigenvalue $\lambda$ for the equal order model problem showing convergence to predicted value -0.2742 with resolution $h=\Delta \sigma$ (left) and domain size $L$ (right)

in the computations. Except for the zero eigenvalue, all other eigenvalues from the discrete problem are in the left half plane. These other discrete eigenvalues approximate the absolute spectrum of the continuous problem. Recall that the absolute spectrum for the limiting problem (20) is given by $(-\infty, b]$ with $b=-c^{2} / 4$. For the equal angle problem, $c=\pi-\theta=\pi / 3$ and $b \approx-0.2742$. It can be seen from Figure 5 that there is good agreement with this analytic result. This is confirmed by resolution studies in $L$ and $\Delta \sigma$ shown in Table 1. Note that as $L$ is further increased, the discrete eigenvalue problem becomes ill-conditioned and gives spurious results in complex pairs, although otherwise the results are not easily recognized as incorrect. This phenomena occurs even for simple problems like (18). It also occurs for large $L$ computations for the mixed order problem discussed below. Resolving this numerical difficulty is of interest but outside the scope of this work.

The stability of travelling waves with different wave speed (obtained by varying the angle $\theta$ shown in Figure 2) are also investigated. All exhibit similar behaviour to that shown above. Other computations with Neumann far field boundary conditions give similar results but with three zero eigenvalues (horizontal translation, vertical shift, and change in wave speed induced by a widening of the curves in the far field). More details of these and other results can be found in [19]. In summary, the computational results of this section give strong evidence that there are no unstable point spectra and thus this equal order travelling wave is spectrally stable. 


\subsection{Stability of travelling waves for the mixed-order model}

For the coupled surface and grain boundary motion, a travelling wave solution was found in $[14,15]$ using an angle formulation for a range of the physical parameter $m$. An additional parameter in the travelling wave is the asymptotic thickness $H$ of the upper grain, although this parameter appears in a different form in the scaling of $[14,15]$. In this section, we consider the stability of these travelling waves. The procedure to derive the discrete, approximate generalized eigenvalue problem is the same as that described in $\S 4.2$ above, except that the derivatives of the exact solution are computed using numerical differentiation of the solution on grid points.

The distribution of eigenvalues for a mixed-order travelling wave is shown in Figure 6. Similar to the simple equal order problem, there is only one zero eigenvalue with associated eigenfunction given by the derivative of the wave. There are no other point spectra. All other eigenvalues are contained in the left half plane which indicates that the travelling wave is stable. It is convincing that the other discrete eigenvalues approximate the absolute spectra of the far field problem (26). The points $P_{1}, P_{2,3}$ of Figure 4 scale to -0.803 and $-0.491 \pm 0.850 i$ respectively using (28) for the problem shown in Figure 6 which has wave speed $c \approx 1.72$. There is good agreement with the results in Figure 6.

Similar results are obtained for a range of values of the parameters $m$ (which governs the junction angle $\theta$ ) and thickness $H$ which is varied by orders of magnitude. With the PDAE formulation, the stability analysis can be applied to the case when one or more of the three curves are not singlevalued. We consider $m=1.995$ when the top right curve is not single-valued. The profile of the three curve near the junction is shown in Figure 7. Again, there is strong evidence that there are no unstable point spectra and thus the mixed order travelling waves are spectrally stable to 2D perturbations.

Figure 8 plots leading eigenvalue (the eigenvalue with largest real part excluding the zero eigenvalue) as a function of the angle $\theta$ between the grain boundary and the free surface. 

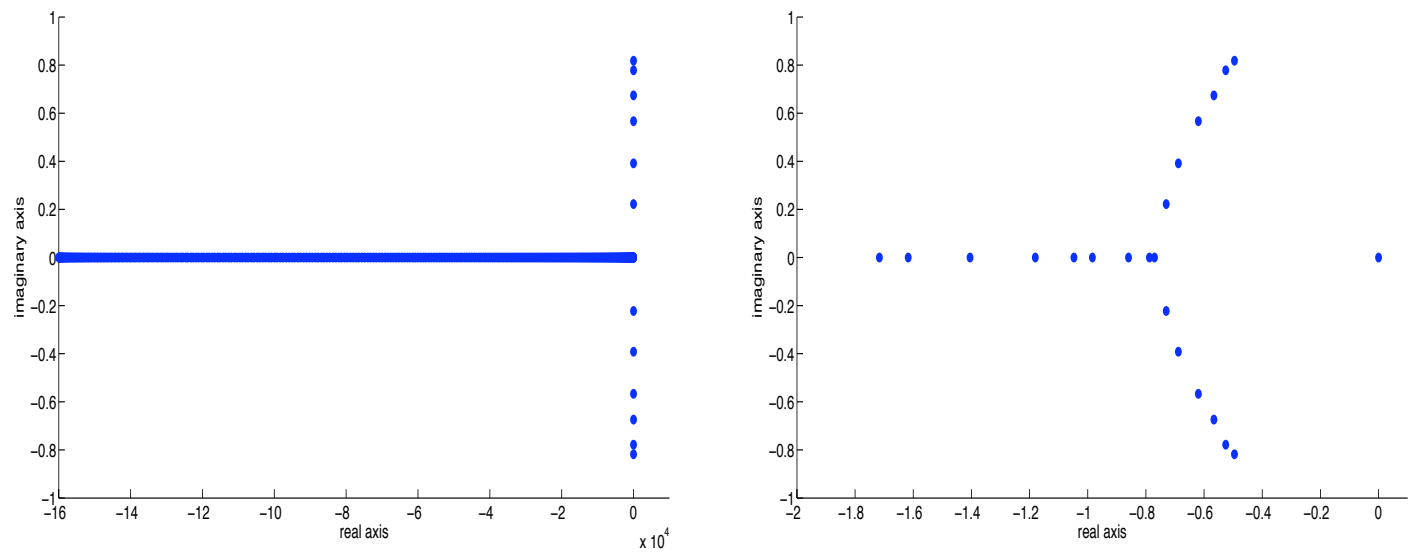

Figure 6: Approximate eigenvalue distribution for the mixed order problem. The computational domain size is $L=20$, discretization $h=\Delta \sigma=0.1$ and the junction angle is $\theta \approx 1.82(\mathrm{~m}=0.5)$. The right picture is a close look of left picture near origin.

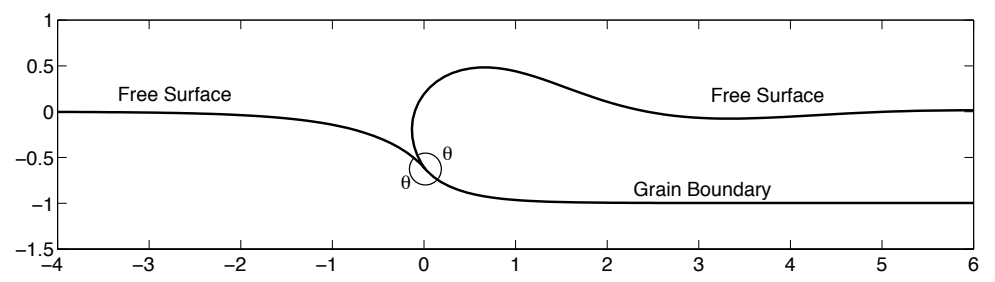

Figure 7: A travelling wave with a non single-valued curve when $\theta=3.07$ $(m=1.995)$. 


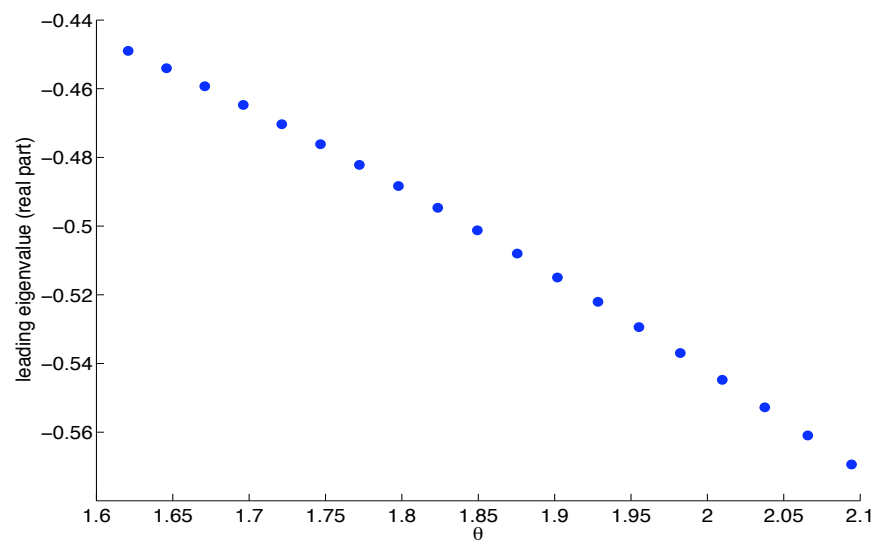

Figure 8: Plot of leading eigenvalue (the eigenvalue with largest real part excluding the zero eigenvalue) as a function of the angle $\theta$ between the grain boundary and the free surface.

\section{$5 \quad$ Stability to 3D perturbations}

In this section, we consider the spectral stability of travelling waves to 3D perturbations. We focus only on the more complicated mixed-order problem. The general approach is similar to that in 2D described above, in which linear perturbations in the direction $z$ orthogonal to the $2 \mathrm{D}$ plane can be described by a Fourier term with wavenumber $\omega$. However, representing surfaces with two parameters and computing surface diffusion evolution is somewhat complicated. Some of the details are given below.

Let $X=(x(\alpha, \beta), y(\alpha, \beta), z(\alpha, \beta))$ represent a surface in 3D. One has the following formula for mean curvature:

$$
H=\frac{G_{11} B_{22}-2 G_{12} B_{12}+G_{22} B_{11}}{2\left(G_{11} G_{22}-G_{12}^{2}\right)}
$$

where

$$
\begin{gathered}
G_{11}=\partial_{\alpha} X \cdot \partial_{\alpha} X, \quad G_{22}=\partial_{\beta} X \cdot \partial_{\beta} X, \quad G_{12}=\partial_{\alpha} X \cdot \partial_{\beta} X \\
B_{11}=\partial_{\alpha}^{2} X \cdot N, \quad B_{22}=\partial_{\beta}^{2} X \cdot N, \quad B_{12}=\partial_{\alpha \beta}^{2} X \cdot N,
\end{gathered}
$$


and $N$ denotes the unit normal given by

$$
N=\frac{\partial_{\alpha} X \times \partial_{\beta} X}{\left\|\partial_{\alpha} X \times \partial_{\beta} X\right\|}
$$

Recall that motion by surface diffusion has normal velocity equal to the surface Laplacian of mean curvature where the surface Laplacian operator is defined by

$$
\Delta_{\text {surf }}=\nabla_{\text {surf }} \cdot \nabla_{\text {surf }}, \quad \nabla_{\text {surf }}=\nabla-N \partial_{n}
$$

where $\partial_{n}=N \cdot \nabla$. Applying the tangential gradient $\nabla_{\text {surf }}$ to a function $H$ defined on a surface $X=(x(\alpha, \beta), y(\alpha, \beta), z(\alpha, \beta))$ gives

$$
\nabla_{\text {surf }} H=g^{11} H_{\alpha} \cdot \partial_{\alpha} X+g^{12} H_{\beta} \cdot \partial_{\alpha} X+g^{21} H_{\alpha} \cdot \partial_{\beta} X+g^{22} H_{\beta} \cdot \partial_{\beta} X
$$

where $g^{i j}$ indicate the components of the inverse matrix of

$$
\left(g_{i j}\right)=\left(\begin{array}{ll}
\partial_{\alpha} X \cdot \partial_{\alpha} X & \partial_{\alpha} X \cdot \partial_{\beta} X \\
\partial_{\alpha} X \cdot \partial_{\beta} X & \partial_{\beta} X \cdot \partial_{\beta} X
\end{array}\right) .
$$

Applying the operator $\nabla_{\text {surf }}$ one more time gives the surface Laplacian of mean curvature $H$ :

$$
\Delta_{\text {surf }} H=\frac{1}{\sqrt{g}}\left(\frac{\partial}{\partial \alpha}\left(g^{11} \sqrt{g} H_{\alpha}+g^{12} \sqrt{g} H_{\beta}\right)+\frac{\partial}{\partial \beta}\left(g^{21} \sqrt{g} H_{\alpha}+g^{22} \sqrt{g} H_{\beta}\right)\right)
$$

where $g=\operatorname{det}\left(g_{i j}\right)$.

With the expressions above, coupled surface and grain boundary motion in $3 \mathrm{D}$ can be described by

$$
\begin{aligned}
& \partial_{t} X_{1} \cdot N_{1}=H_{1}, \\
& \partial_{t} X_{2} \cdot N_{2}=-\Delta_{\text {surf }} H_{2}, \\
& \partial_{t} X_{3} \cdot N_{3}=-\Delta_{\text {surf }} H_{3}, \\
& \partial_{\alpha} X_{i} \cdot \partial_{\alpha}^{2} X_{i}=0 \quad i=1,2,3,
\end{aligned}
$$

where $H_{i}$ represents the mean curvature of curve $i$. The last equation fixes the parametrization $\alpha$ but movement in the other $(\beta)$ tangential direction is not yet fixed. We proceed by considering the surface parametrized as 
$X=(x(\alpha, \beta), y(\alpha, \beta), \beta)$ where $\beta$ becomes the $z$ coordinate. Since the third component is now fixed, we have

$$
\partial_{t} X \cdot N=x_{t} \cdot N_{x}+y_{t} \cdot N_{y}
$$

where $N_{x}, N_{y}$ represent the first two components of the unit normal.

The 2D junction (10) and far field boundary conditions (11) can be extended in a straightforward way to the 3D case. We point out only one of the junction conditions which is associated with the balance of mass flux, the last equation in (10), which becomes

$$
\nabla_{\text {surf }} H_{2} \cdot\left(\partial_{\beta} X_{2} \times N_{2}\right)=\nabla_{\text {surf }} H_{3} \cdot\left(\partial_{\beta} X_{3} \times N_{3}\right)
$$

where the subscripts represent curve indices as before.

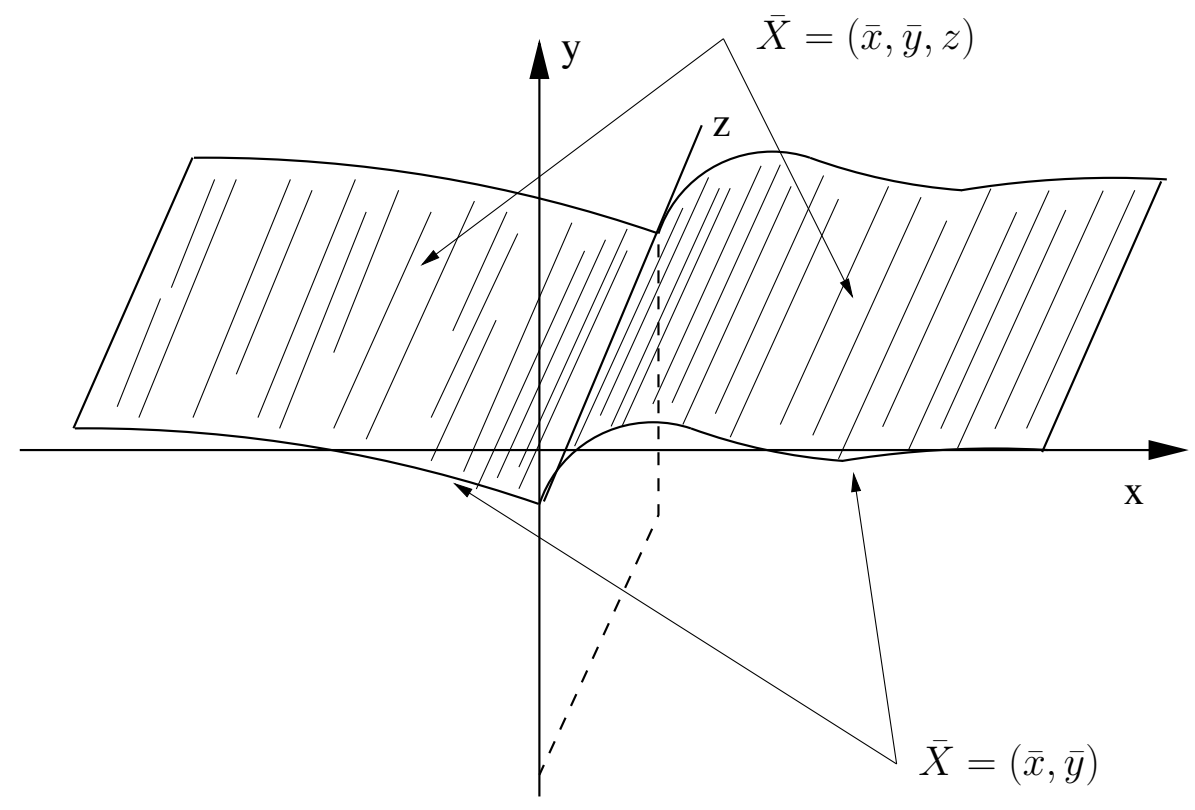

Figure 9: Extension of the 2D travelling wave solution to 3D.

Let $\bar{X}=(\bar{x}(\alpha, t), \bar{y}(\alpha, t))$ represent the travelling wave solution in $2 \mathrm{D}$. A travelling wave solution for the $3 \mathrm{D}$ problem can be expressed in the form of $\bar{X}=(\bar{x}, \bar{y}, z)=(\bar{x}(\alpha, t), \bar{y}(\alpha, t), \beta)$ by extending along the $z$ direction (see Figure 9). 

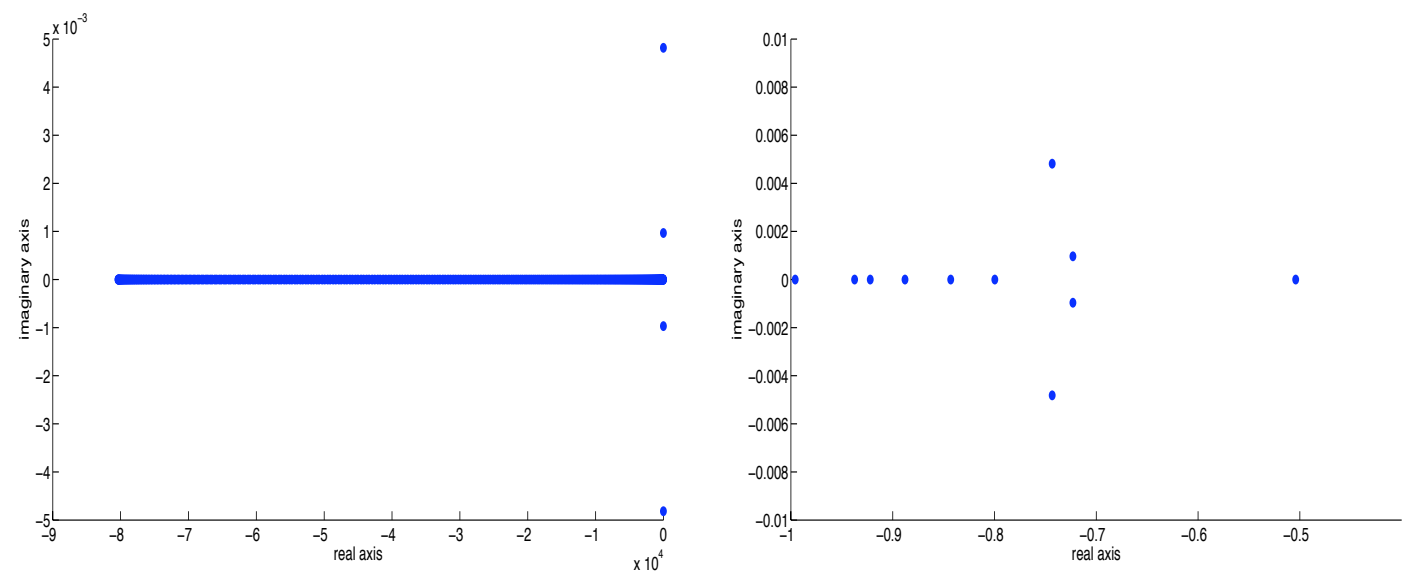

Figure 10: Eigenvalue distribution for the mixed-order problem with 3D perturbations. The computational domain size is $L=20$, discretization $h=\Delta \sigma=0.1$, the junction angle is $\theta=1.82(\mathrm{~m}=0.5)$ and wave number $\omega=1$. The right picture is a closer look of the left picture near origin.

We now repeat the procedure we did for the 2D spectral stability analysis. We consider a small perturbation to the steady state solution in the moving frame

$$
X=\bar{X}+\epsilon e^{\lambda t} e^{i \omega \beta}(\hat{x}(\alpha), \hat{y}(\alpha), 0)
$$

Although the expressions above are complicated, they can be linearized and approximated by finite differences in the same way as the model problem was handled in $§ 4.1$ and lead to a generalized eigenvalue problem. However, due the the complexity of the process, the linearized system was not derived analytically. Instead, the equations were linearized around the travelling wave solution using MATLAB's symbolic toolbox. Example eigenvalue results for $\omega=1$ are shown in Figure 10. A single, leading order point eigenvalue is found. The other discrete eigenvalues fill in an absolute spectra as $L \rightarrow \infty$.

One can see from Figure 10 that all eigenvalues are contained in the left half plane for wave number $\omega=1$. As the wave number is increased, the leading eigenvalue is pushed further to the left. We tested different wave numbers varying from $\omega=0.1$ to $\omega=5$ and the plot of associated leading eigenvalues is shown in Figure 11. Figure 12 plots the leading eigenvalue (the eigenvalue with largest real part) as a function of the angle $\theta$ with wave number $\omega=1$. 


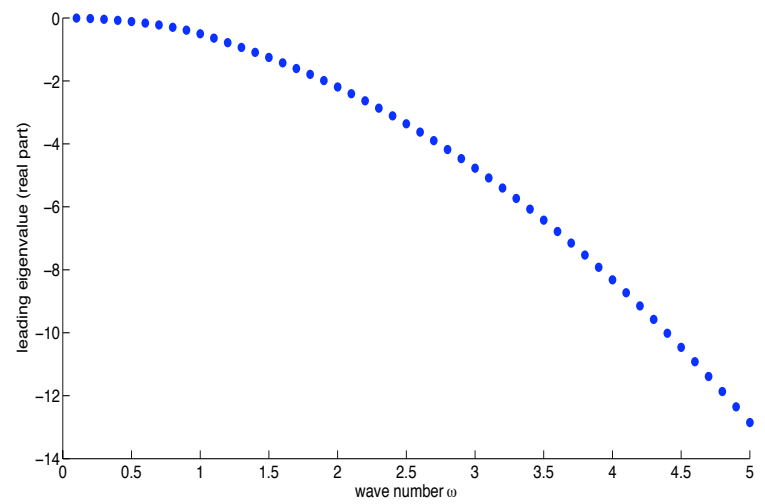

Figure 11: Plot of the leading eigenvalues as a function of wave number for $\omega \in[0.1,5]$.

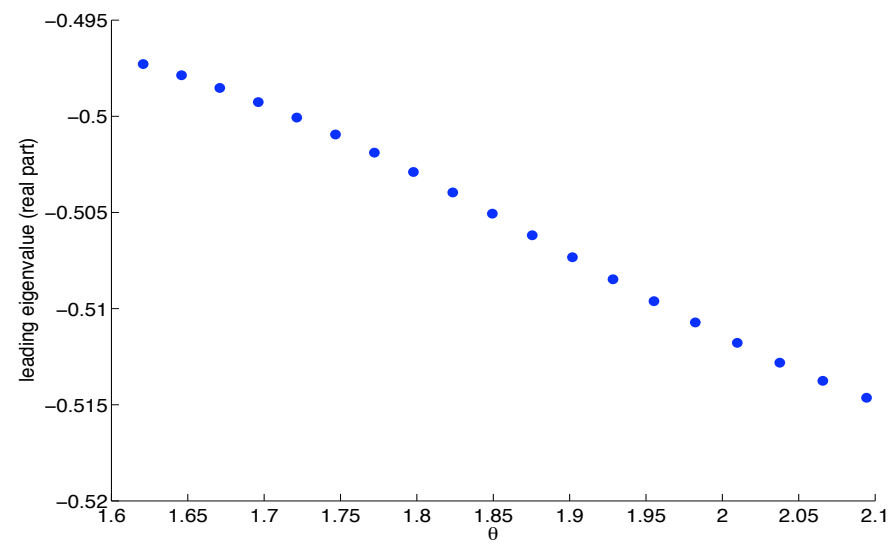

Figure 12: Plot of the leading eigenvalue (the eigenvalue with largest real part) as a function of the angle $\theta$ between the grain boundary and the free surface with wave number $\omega=1$. 
The conclusion on the spectral stability is similar to the $2 \mathrm{D}$ case. There is only one point eigenvalue for each $\omega \neq 0$ and it is in the left half plane. There is strong evidence that the mixed order travelling waves are stable to $3 \mathrm{D}$ perturbations.

\section{Conclusions}

Using a combination of theoretical and numerical methods, strong evidence is given of stability of a well studied, 2D mixed-order travelling wave to both $2 \mathrm{D}$ and $3 \mathrm{D}$ perturbations. The wave is of interest in material science and describes free surface interactions of crystal grains competing during an annealing process. It should be said that this stability is an expected result from physical considerations, but the evidence we provide fills a long standing gap in the study of this process. The novel approach developed in this paper can be used to investigate phenomena in other free surface problems with junctions.

\section{Acknowledgements}

M.B. would like to thank Björn Sandstede for helpful discussions about the effect of domain truncation on spectra of operators. The work of M.B. was partially supported by the NSF under grant DMS-0602891. B.W. would like to thank Amy Novick-Cohen for helpful discussions about the background of the mixed-order problem. The work of B.W. and Z.P. was funded by an NSERC Canada grant.

\section{References}

[1] K. Barmak, M. Emelianenko, D. Golovaty, D. Kinderlehrer, and S. TA'Asan. Towards a Statistical Theory of Texture Evolution in Polycrystals. SIAM Journal on Scientific Computing, 30(6, Sp. Iss. SI):31503169, 2007.

[2] J. W. Barrett, H. Garcke, and R. Nürnberg. On the variational approximation of combined second and fourth order geometric evolution 
equations. SIAM Journal on Scientific Computing, 29(3):1006-1041, 2007.

[3] J. W. Barrett, H. Garcke, and R. Nürnberg. A parametric finite element method for fourth order geometric evolution equations. Journal of Computational Physics, 222(1):441-467, 2007.

[4] Barrett, J.W. and Garcke, H. and Nürnberg, R. Finite Element Approximation of Coupled Surface and Grain Boundary Motion with Applications to Thermal Grooving and Sintering. Universität Regensberg Mathematik Preprint \#11, 2009.

[5] W.-J. Beyn and J. Lorenz. Stability of traveling waves: dichotomies and eigenvalue conditions on finite intervals. Numerical Functional Analysis and Optimization, 20(3):201-244, 1999.

[6] C.-M. Brauner, J. Hulshof, and A. Lunardi. A general approach to stability in free boundary problems. J. Differential Equations, 164(1):16-48, 2000 .

[7] C.-M. Brauner, A. Lunardi, and C. Schmidt-Lainé. Stability of travelling waves with interface conditions. Nonlinear Anal., 19(5):455-474, 1992.

[8] L. Bronsard and F. Reitich. On three-phase boundary motion and the singular limit of a vector-valued Ginzburg-Landau equation. Archive for Rational Mechanics and Analysis, 124(4):355-379, Dec. 1993.

[9] C. Dunn, F. Daniels, and M. Bolton. Transactions of the American Institute of Mining, Metallurgical and Petroleum Engineers, 185:708, 1949 .

[10] M. Furtkamp, G. Gottstein, D. A. Molodov, V. N. Semenov, and L. S. Shvindlerman. Grain boundary migration in fe $\pm 3.5 \%$ Si bicrystals with [001] tilt boundaries. Acta mater., 46:4103-4110, 1998.

[11] H. Garcke and A. Novick-Cohen. A singular limit for a system of degenerate Cahn-Hilliard equations. Advanced Differential Equations, 5:401434, 2000.

[12] G. Gottstein and L. Shvindlerman. Grain Boundary migration in metals: thermodynamics, kinetics, applications. CRC Press, 1999. 
[13] J. Kanel, A. Novick-Cohen, and A. Vilenkin. A traveling wave solution for coupled surface and grain boundary motion. Acta Materialia, 51(7):1981-1989, Apr. 2003.

[14] J. Kanel, A. Novick-Cohen, and A. Vilenkin. Coupled surface and grain boundary motion: a travelling wave solution. Nonlinear Analysis, 59(8):1267-1292, Dec. 2004.

[15] J. Kanel, A. Novick-Cohen, and A. Vilenkin. Coupled surface and grain boundary motion: nonclassical traveling wave solution. Advanced Differential Equations, 9:299-327, 2004.

[16] J. Kanel, A. Novick-Cohen, and A. Vilenkin. A numerical study of grain boundary motion in bicrystals. Acta Materialia, 53(2):227-235, Jan. 2005.

[17] D. Min and H. Wong. A model of migrating grain-boundary grooves with application to two mobility-measurement methods. Acta Materialia, 50(20):5155-5169, Dec. 2002.

[18] W. Mullins. Two-dimensional motion of idealized grain boundaries. Journal of Applied Physics, 27:900-904, 1956.

[19] Z. Pan. Simulation and Analysis of Coupled Surface and Grain Boundary Motion. PhD thesis, University of British Columbia, 2008.

[20] Z. Pan and B. T. Wetton. A numerical method for coupled surface and grain boundary motion. European Journal of Applied Mathematics, 19:311-327, 2008.

[21] B. Sandstede. Stability of travelling waves. Handbook of Dynamical Systems II (B Fiedler, ed.). North-Holland, pages 983-1055, 2002.

[22] B. Sandstede and A. Scheel. Absolute and convective instabilities of waves on unbounded and large bounded domains. Physica D: Nonlinear Phenomena, 145(3-4):233-277, Nov. 2000.

[23] B. Sandstede and A. Scheel. Defects in oscillatory media: toward a classification. SIAM J. Appl. Dyn. Syst., 3(1):1-68 (electronic), 2004.

[24] A. J. Vilenkin, R. Kris, and A. Brokman. Breakup and grain growth in thin-film array. Journal of Applied Physics, 81(1):238-245, 1997. 
[25] H. Zhang and H. Wong. Coupled grooving and migration of inclined grain boundaries: Regime I. Acta Materialia, 50(8):1983-1994, May 2002.

[26] H. Zhang and H. Wong. Coupled grooving and migration of inclined grain boundaries: Regime II. Acta Materialia, 50(8):1995-2012, May 2002. 